Artículos

\title{
Afrontamiento de docentes de enfermería ante la muerte del paciente en unidades de cuidado crítico*
}

\author{
Coping with Nursing Teachers in the Front of Patient Death in Critical Care Units \\ Enfrentamento de docentes de enfermagem frente à morte do paciente em unidades de cuidado crítico
}

\author{
Paula Andrea Duque a \\ Universidad Católica de Manizales, Colombia \\ paduque@ucm.edu.co \\ ORCID: https://orcid.org/0000-0001-7237-6195 \\ Yanier Betancur Manrique \\ Universidad Católica de Manizales, Colombia \\ ORCID: https://orcid.org/0000-0002-1084-1992 \\ Alejandra Franco Galvis \\ Universidad Católica de Manizales, Colombia \\ ORCID: https://orcid.org/0000-0002-1859-2652 \\ Manuela Hoyos Castañeda \\ Universidad Católica de Manizales, Colombia \\ ORCID: https://orcid.org/0000-0001-8941-0783 \\ Esther Edith Valencia Hernández \\ Universidad Católica de Manizales, Colombia \\ ORCID: https://orcid.org/0000-0002-9054-0451
}

DOI: https://doi.org/10.11144/Javeriana.ie22.adem

\section{Resumen:}

Introducción. La muerte es un fenómeno que preocupa a la humanidad, por tanto, es objeto de cuidado por enfermería como parte de las competencias inherentes a la disciplina. Objetivo. Describir el nivel de afrontamiento de las enfermeras frente a la muerte de pacientes en unidades de cuidado crítico. Método. Estudio piloto observacional, de tipo descriptivo transversal, con método de muestreo probabilístico, teniendo en cuenta el número de enfermeras que se desempañaban como docentes de enfermería en el área de cuidado crítico de cuatro universidades de Pereira y Manizales $(\mathrm{n}=30)$. Se aplicó encuesta autoadministrada, elaborada por las investigadoras, con datos sociodemográficos, curriculares y del perfil docente. Para evaluar el nivel de afrontamiento, se utilizó la escala de Bugen de afrontamiento de la muerte, validada al castellano. Resultados. El 100\% de docentes de enfermería tuvo una experiencia cercana a la muerte con algún ser querido, y el 73,3\% no había tenido ninguna preparación como enfermera acerca de la muerte, lo que se puso de manifiesto según la escala de Bugen, donde el $36,8 \%(\mathrm{n}=11)$ de la población puntuó por debajo del percentil 33, el 33,4\% $(n=10)$ de la población por encima del percentil 66 , y el $29,9 \%(n=9)$ puntuó en la zona intermedia. Conclusión. Las enfermeras necesitan mejorar su nivel de afrontamiento ante la muerte, mediante una formación sólida en cuidados integrales al final de la vida y el buen morir, para lograr competencias específicas que puedan ser enseñadas en los actos docentes de manera consciente.

Palabras clave: derecho a morir, adaptación psicológica, cuidados paliativos, atención de enfermería.

\section{Abstract:}

Introduction. The death is a phenomenon, it has always worried humanity, so it is the object of nursing care as part of the inherent competencies of the discipline. Objective. To describe the nurses' level of coping with the death of patients in critical care units. Method. Pilot study observational, descriptive transversal study, the sampling method was probabilistic, taking into account the number of nurses who performed as Nursing teachers in the critical care area of four universities in Pereira and Manizales $(n=30)$. A self-administered survey was applied, prepared by the researchers, with sociodemographic, curricular and teaching profile data, and to assess the level of coping, the Bugen scale of coping with death, validated in Spanish, was used. Results. The 100\% teachers

Notas de autor

a Autora de correspondencia. Correo electrónico: paduque@ucm.edu.co 
had near-death experience with a family member and the $73,3 \%$ had not preparation about the death, which was revelated by the Bugen scale. The 36,8\% (1) of the population scored below the percentile 33 and 33,4\% (2) of the population scored upper the percentile 66 and 29,9\% (3) in the average zone. Conclusion. The nurses need to improve their level of coping with death, through solid training in comprehensive care at the right to die, to achieve specific competencies that can be consciously teach.

Keywords: right to die, adaptation psychological, palliative care, nursing care.

\section{Resumo:}

Introdução. A morte é um fenômeno que preocupa a humanidade, portanto, é objeto dos cuidados de enfermagem no âmbito das competências inerentes à disciplina. Objetivo. Descrever o nível de enfrentamento do enfermeiro frente à morte de pacientes em unidades de cuidado crítico. Método. Estudo piloto observacional, de tipo descritivo transversal, com método de amostragem probabilística, considerando o número de enfermeiros que atuavam como docentes de enfermagem na área de cuidado crítico de quatro universidades de Pereira e Manizales $(\mathrm{n}=30$ ). Inquérito autoaplicado elaborado pelas pesquisadoras foi aplicado, com dados sociodemográficos, curriculares e do perfil docente. Para avaliar o nível de enfrentamento foi utilizada a escala de Bugen de enfrentamento da morte, validada em espanhol. Resultados. $100 \%$ de docentes de enfermagem teve uma experiência de quase morte com um ente querido e $73.3 \%$ não teve preparo nenhum como enfermeira de quase morte, o que foi revelado de acordo com a escala de Bugen, onde 36.8\% $(\mathrm{n}=11)$ da população pontuou por abaixo do percentil 33, 33.4\% $(\mathrm{n}=10)$ da população acima do percentil 66 e 29.9\% (n=9) pontuou na zona intermediaria. Conclusão. O enfermeiro precisa melhorar o seu nível de enfrentamento à morte, por meio de uma sólida formação no cuidado integral no final da vida e o bom morrer para alcançar competências específicas que possam ser ensinadas nos atos docentes de maneira consciente.

Palavras-chave: Direito a morrer, adaptação psicológica, cuidados paliativos, atenção de enfermagem.

\section{Introducción}

La muerte es un fenómeno natural inherente a la condición humana, que comparte aspectos biológicos, sociales, culturales, psicológicos y emocionales $(4,5)$, y es el desenlace de un ser vivo que se había creado a partir de la concepción. En la sociedad occidental, la muerte se concibe como algo extraño e imprevisto, y la mayoría de las veces no está presente en los pensamientos cotidianos, dado el miedo y la incertidumbre que genera, constituyéndose en una fuente de impacto negativo en la vida de las personas $(6,7)$.

La muerte es un fenómeno natural en la vida. Sin embargo, es uno de los que más preocupa a la humanidad, es a la vez un hecho inalterable, ineludible e inexplicable en algunos casos $(8,9)$. Este fenómeno significa, normalmente, dolor y soledad para los que quedan. Por tanto, desde esta perspectiva, no es solo la destrucción de un estado físico y biológico, sino también el fin de un ser en correlación con el otro $(2,3)$.

En el que hacer de la enfermería, el cuidado de la persona al final de la vida y de su familia hace parte de las competencias propias de la profesión $(10,11,12)$, es por eso que ha de ayudar a las personas a enfrentar esta trayectoria, de la vida a la muerte, con una actitud altruista de apoyo que favorezca y promueva el buen morir.

El equipo de enfermería es el más próximo a las personas en las situaciones críticas con inminencia de muerte, es a quien el paciente y las familias buscan cuando precisan de explicaciones, amparo, y cuidado físico y psicológico (13). La enfermera ha de enfrentarse al sufrimiento, la aflicción y los recelos que pueden existir en los diversos momentos que involucran el cuidar, tales acciones exigen no solamente conocimiento de diferentes técnicas instrumentales y enfermedades, sino también actitudes y habilidades para atender los sentimientos de los otros y sus emociones ante el paciente con o sin probabilidad de curación $(13,14)$. Sin embargo, la mayor parte de la formación académica está orientada a la conservación de la vida desde la atención terapéutica, identificándose un vacío de conocimiento en los profesionales de enfermería, porque ante la presencia de la muerte, se alejan, evitando la relación con el paciente y sus allegados por falta de preparación (4). Este es el gran reto no solo para brindar cuidado directo, sino para la docencia de la enfermería, dar respuesta a las necesidades existenciales y facilitar la búsqueda de sentido del quehacer disciplinar $(15,16)$. Teniendo en cuenta que los actos de enseñanza se deben dar desde el conocimiento, puesto que, si el estudiante es guiado adecuadamente en cuanto al afrontamiento a la muerte, logra otorgar mayor valor al sufrimiento, 
abre la brecha al modelo curativo, trasciende el cuidado del ser humano en el fin de vida, y lo reflejara en su futuro manejo profesional.

La respuesta a la muerte ha tenido un significado sociocultural, ligado a conceptos religiosos. En los países occidentales, por ejemplo, el cristianismo anotó que la muerte podría tener un sentido consolador, dado que hay que morir para renacer a la vida eterna, desde otras culturas la muerte se asume con diversos significados que dan una connotación especial de consumación, una forma de ingreso a la plenitud $(17,18)$.

El afrontamiento en las personas es conceptualizado alrededor de las capacidades cognitivas y conductuales para el manejo de situaciones externas y/o internas que son estimadas como desbordantes según los recursos de cada persona para hacerles frente $(19,20)$. En cuanto a la muerte, las personas despliegan un sistema de actitudes que tienen componentes desde lo cognitivo, afectivo y comportamental, es decir lo que piensan de la muerte, cómo se sienten frente a la muerte y qué hacer ante tal suceso de la vida, lo que está determinado por las formas de socializar en las diferentes culturas.

Las estrategias de afrontamiento, según Lazarus y Folkman (19, 21), han de centrarse en el problema para el manejo de las demandas cognitivas que suponen una amenaza con relación a la persona y al manejo de las emociones para la transformación de la incomodidad, y poder manejar los estados emocionales que produce el suceso estresante con el propósito de actuar de manera adecuada. La muerte de los pacientes en cuidado crítico es un proceso complejo y difícil de afrontar, por el desvelo que tiene la enfermera de poner a la persona en las mejores condiciones de vida, sin que se piense en la muerte, es por eso que el afrontamiento de la misma depende de las habilidades cognitivas y recursos personales de las enfermeras, pues un mismo suceso estresante genera en cada persona reacciones muy diversas.

El objetivo del presente estudio fue describir el nivel de afrontamiento que poseen las docentes de enfermería frente a la muerte de los pacientes en los servicios de cuidado crítico.

\section{Método}

Estudio piloto observacional, descriptivo de corte transversal entre agosto de 2018 y marzo de 2019, ejecutado con 30 docentes de las áreas de cuidado critico de enfermería de 4 universidades de las regiones de Manizales y Pereira, Colombia. Se tomó una muestra probabilística, a través de un muestreo aleatorio estratificado, con un índice de confianza del $95 \%$ y un error estimado del $5 \%$, teniendo en cuenta el número de docentes asignados por cada universidad para el acompañamiento de las prácticas en las áreas de cuidado crítico.

Como criterios de inclusión se tuvo en cuenta que las participantes llevaran más de 2 años de experiencia como docentes en áreas de cuidado crítico, y estar contratadas por las universidades en el periodo del estudio; y como criterios de exclusión, enfermeras que estuvieran ausentes al momento de aplicar el instrumento por motivo de incapacidad médica, vacaciones o licencias.

Se realizó la aplicación de encuesta autoadministrada, elaborada por las investigadoras, para variables sociodemográficas y de vivencias de las enfermeras frente a la muerte, con tres preguntas acerca de la preparación que habían tenido frente a la misma, experiencia cercana a la muerte y si había convivido con alguien que está enfermo; las que se plantearon teniendo como fundamento teórico, estudios realizados donde hallaron que a mayor formación frente a la muerte, el afrontamiento era mejor, y conllevaba a brindar una atención más cercana a los pacientes y familiares, también el haber convivido con un familiar en el lecho de muerte, hacia a las enfermeras más sensibles al sufrimiento y con mayor capacidad de afrontamiento (2, 11, 13). La encuesta relacionada fue revisada por 4 expertos, con experiencia en el final de vida y la muerte.

Posteriormente, se aplicó la escala de afrontamiento a la muerte de Bugen, instrumento que fue validado al castellano con una muestra de 916 participantes (580 españoles y 336 cubanos). A los ítems de la escala se les realizaron pruebas de confiabilidad, encontrando un coeficiente alfa de Cronbach de 0,824 (22), lo que evidenció una elevada consistencia interna, de modo que resultó confiable para utilizar en el presente estudio. 
La escala de Bugen mide la competencia para manejar la muerte que tienen las enfermeras y el conocimiento relacionado con sus preparativos $(23,24)$, y está conformada por 30 ítems, con respuestas en formato tipo Likert, cuyas opciones van de 1 ("totalmente en desacuerdo") a 7 ("totalmente de acuerdo"). Se obtiene la puntuación total en competencia para el afrontamiento de la muerte mediante la suma de todos sus ítems, donde se tuvo que invertir los puntajes de los ítems 13 y 24.

Los resultados pueden oscilar de 30 a 210 puntos, donde se interpreta que, a mayor puntuación, mejores competencias para el afrontamiento de la muerte. Cuando puntúan por debajo del percentil 33, se indica bajo afrontamiento, por encima del percentil 66, corresponderían a afrontamiento alto, cuando lo hacen en la zona intermedia, se evidencia un afrontamiento neutro.

La investigación se realizó teniendo en cuenta las normas científicas, técnicas y administrativas contempladas en la resolución Colombiana 8430/1993 (25), que clasifica esta investigación como sin riesgo, al igual que los principios éticos para las investigaciones médicas en seres humanos, postuladas en la declaración de Helsinki. El estudio fue autorizado por el comité de ética de la institución. Con los participantes se explicó de manera individual el consentimiento informado, el objetivo piloto de la investigación, y los beneficios y riesgos, garantizando absoluta confidencialidad.

Para el análisis estadístico de las variables estudiadas se utilizó estadística descriptiva.

\section{Resultados}

TABLA 1.

Características sociodemográficas de las enfermeras participantes del estudio

\begin{tabular}{|c|c|c|c|}
\hline Variable & Categoría & f & $\%$ \\
\hline \multirow{2}{*}{ Género } & Hombre & 4 & $13,3 \%$ \\
\hline & Mujer & 26 & $86,7 \%$ \\
\hline \multirow{4}{*}{ Edad } & 26-30 ańos & 10 & $33,3 \%$ \\
\hline & $31-35$ ańos & 13 & $43,3 \%$ \\
\hline & 36-40 ańos & 1 & $3,3 \%$ \\
\hline & 41-45 ańos & 6 & $20 \%$ \\
\hline \multirow{3}{*}{ Estrato } & Bajo & 1 & $3,3 \%$ \\
\hline & Medio & 14 & $46,7 \%$ \\
\hline & Alto & 15 & $50 \%$ \\
\hline \multirow{4}{*}{ Universidad } & Católica & 11 & $36,7 \%$ \\
\hline & Caldas & 8 & $26,7 \%$ \\
\hline & Libre & 6 & $20 \%$ \\
\hline & Andina & 5 & $16,7 \%$ \\
\hline \multirow{2}{*}{ Ciudad } & Manizales & 19 & $63,3 \%$ \\
\hline & Pereira & 11 & $36,7 \%$ \\
\hline \multirow{4}{*}{ Estado civil } & Casado & 7 & $23,3 \%$ \\
\hline & Soltero & 12 & $40 \%$ \\
\hline & Unión libre & 9 & $30 \%$ \\
\hline & Viudo & 2 & $6,7 \%$ \\
\hline \multirow{3}{*}{ Religión } & Católico & 26 & $86,7 \%$ \\
\hline & Cristiano & 3 & $10 \%$ \\
\hline & Otro & 1 & $3,3 \%$ \\
\hline \multirow{4}{*}{ Ańos de experiencia } & $2-6$ años & 16 & $53,3 \%$ \\
\hline & 7-11 ańos & 7 & $23,3 \%$ \\
\hline & 12-16 ańos & 5 & $16,6 \%$ \\
\hline & $>17$ ańos & 2 & $6,6 \%$ \\
\hline \multirow{4}{*}{ Nivel de formación } & Pregrado & 7 & $23,3 \%$ \\
\hline & Especialización & 15 & $50 \%$ \\
\hline & Maestría & 6 & $20 \%$ \\
\hline & Doctorado & 2 & $6,7 \%$ \\
\hline
\end{tabular}

TABLA 2.

Análisis descriptivo de la encuesta establecida para vivencias de las enfermeras frente a la muerte

\begin{tabular}{|c|c|c|c|}
\hline Variable & Categoría & $f$ & $\%$ \\
\hline ¿Como profesional, & Sí & 8 & $26,7 \%$ \\
\hline $\begin{array}{l}\text { ha tenido } \\
\text { preparación acerca de } \\
\text { la muerte? }\end{array}$ & No & 22 & $73,3 \%$ \\
\hline $\begin{array}{l}\text { ¿Ha tenido alguna } \\
\text { experiencia cercana } \\
\text { de muerte con algún } \\
\text { ser querido? }\end{array}$ & Sí & 30 & $100 \%$ \\
\hline ¿Convive con alguien & Sí & 5 & $16,7 \%$ \\
\hline que está enfermo? & No & 25 & $83,3 \%$ \\
\hline
\end{tabular}




\section{Escala de Bugen afrontamiento a la muerte}

El 36,8\% $(n=11)$ de la población ha puntuado por debajo del percentil 33. El 33,4 $(n=10)$ de la población ha puntuado por encima del percentil 66 , y el $29,9 \%(n=9)$ en la zona intermedia.

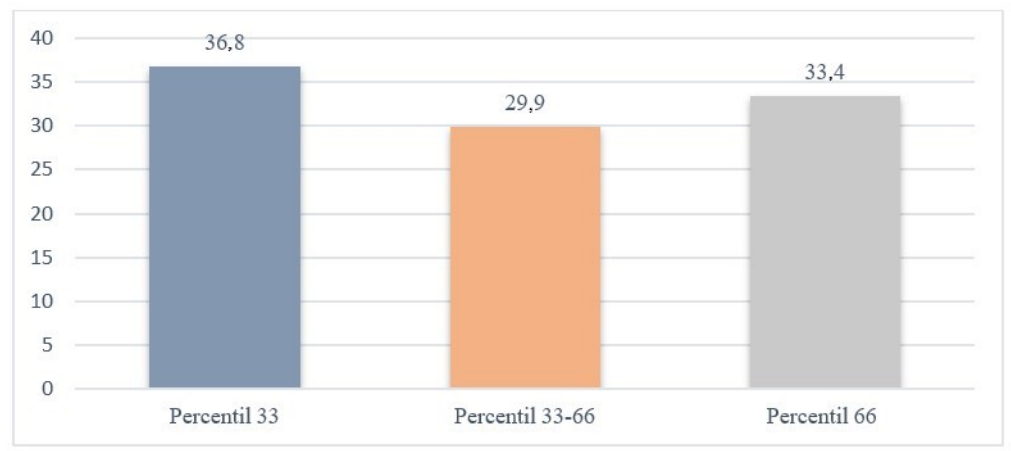

FIGURA 1.

Nivel de Afrontamiento a la Muerte según la Escala de Bugen Fuente: elaboración propia

En cuanto a la media total del afrontamiento basado en la escala de Bugen, se obtuvo un puntaje de 154 puntos con un mínimo de 103 y un máximo de 188.

TABLA 3.

Afrontamiento a la muerte por ítems según escala de Bugen

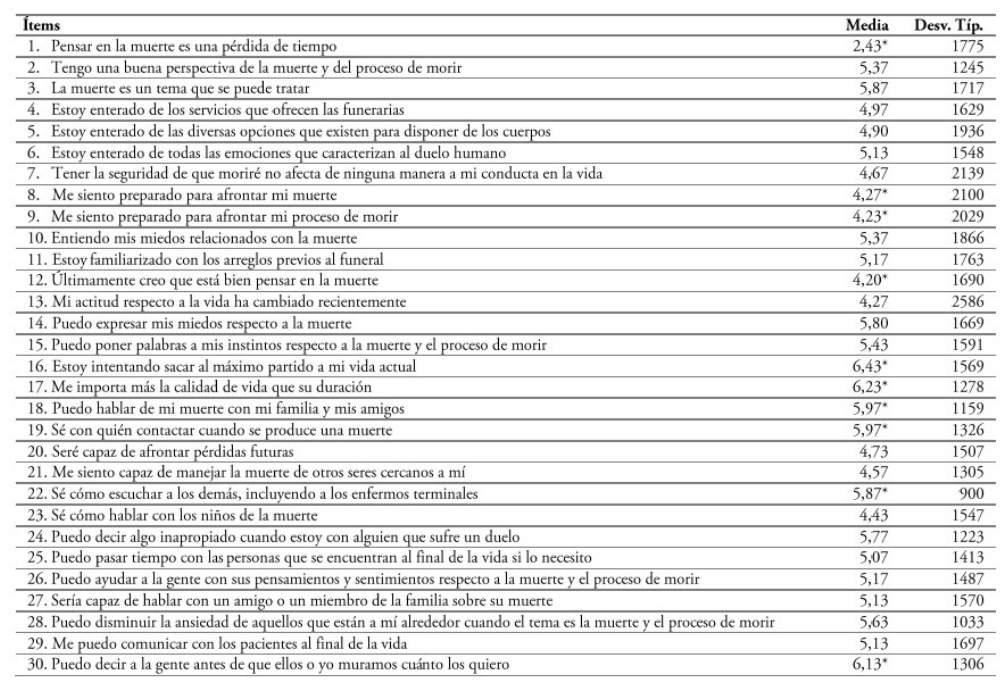

* Datos que evidencian los ítems para afrontamiento bajo, medio y alto Fuente: elaboración propia

En cuanto a los promedios relevantes obtenidos de acuerdo con cada pregunta, se realizó la siguiente clasificación: afrontamiento bajo, neutro y alto.

En el afrontamiento bajo se encontró el ítem 1, "Pensar en la muerte es una pérdida de tiempo" con una media de 2,43, lo cual significa que la muerte no es una pérdida de tiempo. En el afrontamiento neutro se encontraron los ítems 8 "Me siento preparado para afrontar mi muerte", el ítem 9 "Me siento preparado para afrontar mi proceso de morir", y el ítem 12 "Últimamente creo que está bien pensar en la muerte", lo cual significa que estos ítems se centran en el proceso que cada profesional tiene respecto a la muerte.

En el afrontamiento alto se encontraron los ítems 16 "Estoy intentando sacar al máximo partido a mi vida actual", el ítem 17 "Me importa más la calidad de vida que su duración”, y el 30 "Puedo decir a la gente antes de que ellos o yo muramos cuanto los quiero", donde parece que el profesional puede expresar sus emociones frente a las personas que le son significativas. 


\section{Discusión}

La muerte establece un impacto emocional significativo en las enfermeras, quienes experimentan respuestas humanas de temor y angustia, que en ocasiones se traducen en distanciamiento o evasión por el fracaso que sienten ante la no recuperación de la persona cuidada. Una revisión de literatura realizada evidenció en diferentes culturas cómo el personal de enfermería que se encuentra en contacto con la muerte experimenta ansiedad e intranquilidad (26). La muerte, aunque se conceptualice como un fenómeno natural, no deja de ser una experiencia impactante, además de ser una realidad que no siempre es aceptada y nunca deja de ser un hecho temido por estudiantes y/o profesionales de la salud con varios años de experiencia (27). La manera de afectación puede ser física y emocional, provocando dolor ante la pérdida lo que representa una fuerte perturbación en el quehacer de las enfermeras $(16,28)$.

Un hallazgo significativo en el presente estudio fue el de los años de experiencia de las enfermeras, que se encuentra entre 4 y 20 años, donde no habían tenido preparación para afrontar la muerte de manera eficaz, lo que da a entender que este proceso se vive de manera individual. Se asemeja a un estudio realizado, en el cual se concluyó que, aunque las enfermeras perciben la necesidad de respetar la dignidad del paciente, brindar cuidados dirigidos al bienestar y favorecer la inclusión de la familia, existe una falta de conocimientos de las enfermeras de cuidado crítico en la atención que se debe brindar al final de la vida (29). Las experiencias, como circunstancias o acontecimientos vividos por una persona, constituyen elementos importantes en el trabajo de enfermería (30), por lo que los profesionales más experimentados tienen más habilidad en abordar temas relacionados con las decisiones sobre la muerte, y esto se valora positivamente por parte del resto del equipo, donde los profesionales más jóvenes expresan sus opiniones con mayor dificultad.

En el presente estudio las enfermeras profesaron tener creencias religiosas fundamentadas en el catolicismo, como un acto necesario ligado a la espiritualidad, expresado como la sensibilidad o el apego a los valores religiosos en contraposición con el interés material o mundano, lo que indica una atención desde la dimensión espiritual por enfermería a las personas al final de la vida, aspecto importante si se tiene en cuenta que en este campo se cuida la persona en sus diferentes dimensiones. Un estudio realizado en Inglaterra demostró las implicaciones que tiene para el paciente, en términos de calidad de vida, cuando la enfermera no puede atender sus necesidades espirituales (31).

La espiritualidad se refiere a la conciencia de un Yo interior, y a la propensión a construir significados a través de la sensación de estar unido a dimensiones que trascienden al ego, y que pueden ser experimentadas en niveles intrapersonales y transpersonales, lo que reitera la necesidad de atención por parte del equipo de salud desde esta dimensión (32), y otorga sentido al cuidado por ser un acto de atención con del otro, situado en medio de la interacción de costumbres y creencias, las cuales son una forma de conocimiento interiorizado, que se traduce en la representación de mirar y sentir el mundo.

En un estudio realizado en el área de cuidados paliativos, las enfermeras relataron que, durante la práctica profesional, aprendieron a valorar la importancia del cuidado individualizado al final de la vida, ya que durante el pregrado tuvieron poco contacto con la temática y con ese tipo de pacientes. Resaltaron la necesidad de experiencias prácticas para el desarrollo de herramientas útiles, para que el cuidado sea de calidad. Además, comprendieron la importancia del buen morir, y se verificó que en los diferentes discursos dejan claro que fueron las experiencias vividas las que modificaron sus filosofías de vida (33). Resulta necesario que las enfermeras sean sensibles a las demandas del paciente y su familia, una vez que en cada etapa de la enfermedad los deseos se modifican, especialmente durante la fase terminal. Al respecto, un estudio realizado en Lima, con pacientes en cuidados paliativos, concluyó que es relevante la actuación de enfermería en la mitigación del dolor (34).

En el presente estudio el $73,3 \%$ no ha tenido ninguna preparación como profesional acerca de la muerte, y el 26,7\% restante sí la ha tenido, lo que se relaciona con un estudio realizado en Brasil (35), donde se evidenció debilidad en la capacitación de las enfermeras con respecto al proceso de muerte, identificando la necesidad 
de implementar estrategias de afrontamiento, educación continua e intercambio de experiencias. Por tanto, es evidente la necesidad de preparar a las enfermeras en el cuidado al final de la vida, la muerte y su proceso.

Con relación al afrontamiento a la muerte, el presente estudio demostró que en los profesionales de enfermería frente a la muerte es del $36,7 \%$, puntuando por debajo del percentil 33 como afrontamiento bajo, 33,4\% puntuando por encima del percentil 66 como afrontamiento alto, y 29,9\% como neutro con un percentil entre el 33-66. Otros estudios realizados enfatizan la necesidad de recibir conocimientos y la actitud que se ha de asumir en cuanto a la muerte de los pacientes $(14,36)$.

En la indagación pregunta por pregunta, se identificó que uno de los ítems más bajos es el 1, "Pensar en la muerte es una pérdida de tiempo", con un promedio de 2,43. La muerte en el ámbito hospitalario, y desde el punto de vista biomédico, se percibe más como un fracaso técnico que como un fenómeno natural del ser humano.

Por otra parte, una adecuada práctica de enfermería al final de la vida se basa en respetar la dignidad de los pacientes y facilitar un buen morir. Sin embargo, las enfermeras de cuidado crítico tienden a tecnificar sus habilidades y destrezas, por lo que se han especializado en adelantarse y controlar, con cuidados únicamente físicos, las situaciones que comprometen la vida del enfermo, pero no en administrar cuidados paliativos o de bienestar (17).

El ítem 8 "Me siento preparado para afrontar mi muerte", con un promedio neutro de 4,27, se relaciona con un estudio realizado a docentes que laboran en unidades de cuidado crítico, sobre el sentido de la vida y la muerte en Chiclayo, Perú, el cual expresa que, desde la creación del hombre, la relación que establece con otros seres de su misma especie le imprime el carácter de ser social por naturaleza (37). Puesto que habita en un entorno donde no está solo, sino que se relaciona con otras personas, con costumbres, intereses e ideas propias, con pensamientos llenos de sueños, proyectos, y metas que desean cumplir durante su vida, se desarrolla como ser humano útil para su sociedad.

Otro ítem que presentó un promedio neutro de 4,23 está relacionado con la pregunta 9, "Me siento preparado para afrontar mi proceso de morir", lo cual demuestra que la enfermera, antes de graduarse, es una persona que día a día se enfrenta a retos, entre ellos la muerte, sin sentirse preparada para afrontar el proceso de la misma. Un estudio realizado en México, relacionado con el impacto psicosocial en enfermeras que cuidan personas al final de la vida, evidenció que la manera de ver la muerte proviene de lo cultural (38), se llora y teme y a la vez se conmemora y trivializa, pero siempre suscita emociones intensas aun cuando se le termina aceptando como algo natural y como una forma de transitar a otro plano de existencia en otra dimensión.

Por otro lado, hay diferentes ítems en el afrontamiento alto, como el 16, "Estoy intentando sacar al Máximo partido a mi vida actual", con un promedio de 6,43; y el 18, "Puedo hablar de mi muerte con mi familia y mis amigos", con un promedio de 5,97, donde se identifica que el ítem más alto se relaciona con la vida íntima y personal de la enfermera, que brinda sentido a su existencia. A parte de las concepciones de la muerte, surgidas de las experiencias vividas, enfrentar la muerte es un reto ético importante, porque las enfermeras se encuentran inmersas dentro de un sistema que tiene como objetivo principal la restauración de la salud, por lo que la muerte se ve como una decepción, por lo cual no es sorprendente que los profesionales de enfermería, al enfrentarse a la muerte, se muestren ansiosos, y tengan dificultades para afrontar los sentimientos que afloran hacia la muerte (39).

El ítem 29, "Me puedo comunicar con los pacientes al final de la vida", con un promedio de 5,13, significa que los profesionales cuidan, escuchan y brindan bienestar al paciente, y se complementa con una investigación realizada en Turquía, donde se plantea que la comunicación contempla un gran número de actitudes personales, que, a su vez, puede hacer que las enfermeras se sientan incómodas al brindar atención a los pacientes moribundos (40).

Como estrategias de afrontamiento, las enfermeras utilizan de forma predominante el distanciamiento, la evitación y la disociación emocional. Estas estrategias aparecen recogidas en otro estudio realizado en Turquía con enfermeras de urgencias y cuidados críticos (41), donde el distanciamiento es llevado a cabo por las 
enfermeras para que el sufrimiento ante la pérdida del paciente sea menor, esto hace que la enfermería se centre más en el cuidado físico que el cuidado emocional del paciente moribundo, haciendo así que tanto la implicación emocional con el paciente como la repercusión emocional en la enfermera sea mucho menor (34).

\section{Conclusiones}

A partir de los datos y la manera como las enfermeras acogen la muerte, se evidenció debilidad en la preparación para el afrontamiento de esta, ya que se observó que las enfermeras con más trayectoria presentan dificultad en expresar sus sentimientos ante el final de la vida, y las que tenían menos experiencia tenían temor a tomar decisiones, por lo que quedaban distantes de la situación. Así mismo, las vivencias de las enfermeras mostraron que no han recibido preparación, en su mayoría, para enfrentar la muerte, aun cuando son profesionales que se desempeñan en áreas de cuidado crítico y están encargadas de enseñar en pregrado de enfermería, lo que pone en evidencia el miedo que se tiene a la muerte y la necesidad de mantener la vida en los pacientes.

El afrontamiento en el estudio puntuó por debajo del percentil 33, lo que significa que es bajo, e que invita a reflexionar sobre el rol del enfermero y el afrontamiento que ha de tener frente a la muerte, no solo con el paciente, los familiares y allegados, sino también con el estudiante y consigo mismo como cuidador. Es decir, la muerte ha de manejarse con el conocimiento, las emociones y el actuar adecuado, puesto que avanzar en el afrontamiento a la muerte es comprender la importancia del cuidado a la persona al final de la vida, con actos centrados en el bienestar y no en la curación.

\section{Referencias}

1. Thiele T, Dunsford J. Nurse leaders' role in medical assistance in dying: A relational ethics approach. Nurs Ethics [Internet]. 2017;26(4). https://doi.org/10.1177/0969733017730684

2. Celma AG, Strasser G. El proceso de muerte y la enfermería. Physis Rev Saúde Coletiva. 2015;25(2):485-500.

3. Coyne P, Mulvenon C, Paice JA. American Society for Pain Management Nursing and Hospice and Palliative Nurses Association Position Statement: Pain Management at the End of Life. Pain Manag Nurs. 2018;19(1):3-7. http s://doi.org/10.1016/j.pmn.2017.10.019

4. Freitas TLL, Banazeski AC, Eisele A, de Souza EN, Bitencourt, JV, Souza SS. La visión de la Enfermería ante el Proceso de Muerte y Morir de pacientes críticos: una revisión integradora. Enferm Global. 2016;5(41):322-334.

5. Ganz FD, Sapir B. Nurses' perceptions of intensive care unit palliative care at end of life. Nurs Crit Care [Internet]. 2019;24:141-148. https://www.doi.org/10.1111/nicc.12395

6. Ong KK, Ting KC, Chow YL. The trajectory of experience of critical care nurses in providing end-of-life care: A qualitative descriptive study. J Clin Nurs [Internet]. 2018;27:257-268. https://doi.org/10.1111/jocn.13882

7.Zheng R, Lee SF, Bloomer MJ. How new graduate nurses experience patient death: a systematic review and qualitative meta-synthesis. Int J Nurs Stud [Internet]. 2016;53:320-330. https://doi.org/10.1016/j.ijnurstu.2015.09.013

8. Sanchís J. Afrontamiento del miedo a la muerte [tesis doctoral]. [Madrid]: Departamento de Psicología Evolutiva y de la Educación, Universidad Complutense de Madrid; 2017.

9. Díaz MC, Juarros N, García B, Sáez C. Study on anxiety in intensive care nursing professionals facing the process of death. Enferm Global. 2017;16(1): 256-265.

10. Thiele T, Dunsford J. Nurse leaders' role in medical assistance in dying: A relational ethics approach. Nurs Ethics [Internet]. 2017;26(4). https://doi.org/10.1177/0969733017730684

11. Fernández JA, García L, García ML, Fernández AI, Lozano T. Actitudes y afrontamiento ante la muerte en el personal de Enfermería. TEYS. 2017;2(1):27-33. 
12. Brooks LA, Bloomer MJ, Manias E. Culturally sensitive communication at the end-of-life in the intensive care unit: A systematic review. Aust Crit Care [Internet]. 2018;32(6):516-523. https://doi.org/10.1016/j.aucc.2018.07. 003

13. Freitas T, Banazeski AC, Eisele A, Souza EN, Bitencourt JV, Souza SSD. La visión de la Enfermería ante el Proceso de Muerte y Morir de pacientes críticos: una revisión integradora. Enferm. Glob. 2016;15(41):322-334. Disponible en: http://scielo.isciii.es/scielo.php?script=sci_arttext\&pid=S1695-61412016000100015\&lng=es

14. Marchan S. Afrontamiento del profesional de enfermería ante la muerte de pacientes, en unidades de paliativos y oncología. Nure Inv. 2016;13(82). Disponible en: https://www.nureinvestigacion.es//OJS/index.php/nure/ar ticle/view/789

15. Edo-Gual M, Monforte C, Tomás J. Afrontar el sufrimiento y la muerte: desafíos para el cuidado en el siglo XXI. Enferm Clin. 2015;25(1):42-44.

16. Ferrell B, Malloy P, Mazanec P, Virani R. CARES: AACN's New Competencies and Recommendations for Educating Undergraduate Nursing Students to Improve Palliative Care. Journal Prof Nurs [Internet]. 2016;32(5):327-333. https://doi.org/10.1016/j.profnurs.2016.07.002

17. Krystallidou D, Devisch I, Van de Velde D, Pype, P. Understanding patient needs without understanding the patient: the need for complementary use of professional interpreters in end-of-life care. Med. Health Care Philos. 2017;20(4):477-481. https://doi.org/10.1007/s11019-017-9769-y

18. Mazzetti C. Nombrar la muerte. Aproximaciones a lo indecible. Andamios [Internet]. 2017;14(33). http://doi.o $\mathrm{rg} / 10.29092 / \mathrm{uacm} . \mathrm{v} 14 \mathrm{i} 33.545$

19. Antoniazzi A, Dell'Aglio D, Bandeira D. O conceito de coping: uma revisão teórica. Estud. Psicol. (Natal) [Internet]. 1998;3(2):273-294. https://doi.org/10.1590/S1413-294X1998000200006

20. Viñas F, González M, García Y, Malo S, Casas A. Coping strategies and styles and their relationship to personal wellbeing in a sample of adolescents. An Psychol. 2014;31(1):226-233.

21. Henao ÁM, Quiñonez MA. Afrontamiento de las enfermeras ante la muerte del paciente en la Unidad de Cuidado Intensivo Pediátrico. Enferm Intensiva [Internet]. 2018;30(4):163-169. https://doi.org/10.1016/j.enfi.2018.1 0.005

22. Schmidt J. Validación de la versión española escala de Bugen de afrontamiento de la muerte y Perfil revisado de actitudes hacia la muerte: Estudio comparativo y transcultural [tesis doctoral]. [Granada]: Universidad de Granada, Escuela Universitaria de Ciencias de la Salud; 2007.

23. Bugen LA. Coping: Effects of death education. J Death Dying. 1981;11(2):175-183.

24. Bugen LA. Human grief: a model for prediction and intervention. Am J Orthopsychiatry. 1977;47:196-206.

25. Ministerio de Salud de Colombia. Resolución 8430 de 1993. Bogotá: Ministerio de Salud; 1993.

26. Nia HS, Lehto RH, Ebadi A, Peyrovi H. Death anxiety among nurses and health care professionals: A review article. Int J Community Based Nurs Midwifery. 2016;4(1):2-10.

27. Orozco MÁ, Tello GO, Sierra A, Gallegos RM, Xeque ÀS, Reyes BL, Zamora A. Experiencias y conocimientos de los estudiantes de enfermería, ante la muerte del paciente hospitalizado. Rev enfermería universitaria. 2016;10(1):8-13.

28. Álvarez D. La experiencia de las enfermeras ante la muerte [tesis doctoral]. [Madrid]: Revisión Narrativa, Universidad Autónoma de Madrid; 2016.

29. Piedrafita AB. Percepciones, experiencias y conocimientos de las enfermeras sobre cuidados paliativos en las unidades de cuidados intensivos. Rev Enferm Intens. 2015;26(4)121-168.

30. Hunt K, Bernal J, Worth R, Shearn J, Jarvis P, Jones E, et al. End-of-life care in intellectual disability: a retrospective cross-sectional study. BMJ Support Palliat [Internet]. 2019 oct. 16. https://doi.org/10.1136/bmjspcare-2019001985

31. O'Brien MR, Kinloch K, Groves KE, Jack BA. Meeting patients' spiritual needs during end-of-life care: A qualitative study of nurses' and healthcare professionals' perceptions of spiritual care training. J. Clin. Nurse [Internet]. 2019;28(1-2):182-189 https://doi.org/10.1111/jocn.14648 
32. Daudt HD, Archangelo M, Duquette D. Spiritual care training in healthcare: Does it really have an impact? Palliat Support Care [Internet]. 2019.17(2):129-137. https://doi.org/10.1017/S1478951517001134

33. Griffith S. Prepared for end-of-life care: a concept analysis. Int. J. Palliat. Nurs [Internet]. 2018;24(8):399-410. h ttps://doi.org/10.12968/ijpn.2018.24.8.399

34. Fhon JRS, Silva LM, Rodríguez RAP, Carhuapoma AME. Percepción de las enfermeras sobre cuidados paliativos. Experiencia con pacientes oncológicos. Rev Iberoam Educ Invest Enferm. 2018;8(3):28-36.

35. Grams S, Kahl C, Cunha K, Koerich C, Santos T, Lorenzini A. The process of death and dying. Challenges in nursing care for patients and family members. Rev Rene [Internet]. 2017;18(4):528-35. https://doi.org/10.43 28/JCAM.4949

36. Marchan S. Afrontamiento de los profesionales de enfermería en el duelo neonatal [trabajo de pregrado]. [Coruña]: Facultade de Enfermaría e Podoloxía, Universidade da Coruña; 2017.

37. Torres MD, Zapata RA. Sentido de vida/muerte de los docentes de la Escuela de Enfermería, que laboran en Unidades Críticas. USAT. Chiclayo. Rev Acc Cietnavol. 2015;2(2):34-45.

38. Pérez-Vega ME, Cibanal JL. Impacto Psicosocial en Enfermeras que brindan Cuidados en Fase Terminal. Rev. Cuidarte. 2016;7(1):1210-1218.

39. Celma AG, Strasser G. un enfoque relacional. Reflexiones teóricas en torno a la atención frente a la muerte. Physis Rev Saúde Coletiva. 2015;25(2):485-500

40. Ozcelik H, Aksoy F, Sonmez E, Fadiloglu C. Attitudes to death of nurses in Turkey and factors affecting them. MedCrave. 2018;2(6):1-7.

41. Şahin DS, Önal Ö, inanç BB. Attitudes of nurses working emergency and intensive care units toward good death and death anxiety. J. Clin. Anal. Med. 2017;7:75-79.

\section{Notas}

* Artículo de investigación. Proyecto patrocinado por la Universidad Católica de Manizales. Limitación del estudio: el tamaño de la muestra se redujo, dado que algunos de los docentes de las universidades, no alcanzaron a participar en el estudio piloto.

\section{Licencia Creative Commons CC BY 4.0}

Cómo citar este artículo: Duque PA, Betancur Manrique Y, Franco Galvis A, Hoyos Castañeda M, Valencia Hernández EE. Afrontamiento de docentes de enfermería ante la muerte del paciente en unidades de cuidado crítico. Investig Enferm Imagen Desarr. 2020;22. https://doi.org/10.11144/Javeriana.ie22.adem 